\title{
A plane quartic curve with twelve undulations
}

\author{
By W. L. ĖdaE.
}

1. The pencil of quartic curves

$$
x^{4}+y^{4}+z^{4}+\lambda\left(y^{2} z^{2}+z^{2} x^{2}+x^{2} y^{2}\right)=0,
$$

where $x, y, z$ are homogeneous coordinates in a plane, was encountered by Ciani [Palermo Rendiconti, Vol. 13, 1899] in his search for plane quartic curves that were invariant under harmonic inversions. If $x, y, z$ undergo any permutation the ternary quartic form on the left of (1) is not altered; nor is it altered if any, or all, of $x, y, z$ be multiplied by -1 . There thus arises an octahedral group $G$ of ternary collineations for which every curve of the pencil is invariant. Since (1) may also be written

$$
(\lambda+2)\left(x^{2}+y^{2}+z^{2}\right)^{2}+(\lambda-2)(x+y+z)(y+z-x)(z+x-y)(x+y-z)=0
$$

the four lines

$x+y+z=0, \quad y+z-x=0, \quad z+x-y=0, \quad x+y-z=0$

are, as Ciani pointed out, bitangents, at their intersections with the conic $C$ whose equation is $x^{2}+y^{2}+z^{2}=0$, to every quartic of the pencil. The 16 base points of the pencil are thus all accounted forthey consist of these eight contacts counted twice-and this set of points must of course be invariant under $G$. Indeed the 4 ! collineations of $G$ are precisely those which give rise to the different permutations of the four lines (2), a collineation in a plane being determined when any four non-concurrent lines and the four lines which are to correspond to them are given. The quadrilateral formed by the lines (2) will be called $q$.

There are certain curves (1) which have special properties, notably the following:-

$$
\begin{aligned}
& \lambda=-2: \text { the quadrilateral } q \text {. } \\
& \lambda=2 \text { : the repeated conic } C \text {. } \\
& \lambda=-1: \text { the two conics }\left(x^{2}+\omega y^{2}+\omega^{2} z^{2}\right)\left(x^{2}+\omega^{2} y^{2}+\omega z^{2}\right)=0 \text {, } \\
& \text { where } \omega^{3}=1 \text {. Each conic is both inpolar and out- } \\
& \text { polar to the other, as well as to } C \text {. } \\
& \lambda=\infty \text { : a curve with three biflecnodes. } \\
& \lambda=\quad \text { either root of } \lambda^{2}+3 \lambda+18=0 \text { : a Klein quartic, } \\
& \text { admitting a group of } 168 \text { collineations. } \\
& \lambda=0: \text { Dyck's curve, admitting a group of } 96 \text { collineations. }
\end{aligned}
$$


One property of this latter curve is that its inflections coincide in pairs at twelve undulations, at each of which the tangent has fourpoint contact with the curve.

It is the purpose of this note to point out that there is another curve belonging to the pencil which also has its inflections coinciding in pairs at twelve undulations, namely the curve for which $\lambda=3$.

2. We consider then the curve $Q$ whose equation is

$$
\Sigma \equiv x^{4}+y^{4}+z^{4}+3\left(y^{2} z^{2}+z^{2} x^{2}+x^{2} y^{2}\right)=0 .
$$

Since $\Sigma$ can be written in the form

$$
(2 i y+z-x)(2 i y+z+x)(2 i z+x-y)(2 i z-x-y)+5(y-i z)^{4} \text {, }
$$

where $i^{2}=-1$, it follows that the four intersections of $Q$ with the line $y=i z$ are undulations, and that the equations of the respective tangents are

$2 i y+z-x=0, \quad 2 i y+z+x=0, \quad 2 i z+x-y=0, \quad 2 i z-x-y=0$.

Another tetrad of undulations, on the line $y=-i z$, is obtained by changing the sign of $i$, and the equations of the tangents there also. Further information is yielded by cyclic permutation of $x, y, z$; and the outcome is as follows.

The curve $Q$ has 12 undulations, four on each of the lines

$$
\begin{aligned}
& y=i z, \quad z=i x, \quad x=i y, \\
& y=-i z, \quad z=-i x, \quad x=-i y \text {. }
\end{aligned}
$$

The undulations, together with the vertices of $\Delta$, the diagonal triangle of $q$, make up the complete set of intersections of these six lines. The coordinates of the undulations, with the equations of the respective tangents, are

$$
\begin{array}{rlll}
(i, \quad 1,-1): & 2 i x=z-y, & (-i, 1,-1): & 2 i x=y-z, \\
(-1, i, \quad 1): & 2 i y=x-z, & (-1,-i, 1): \quad 2 i y=z-x, \\
(1,-1, \quad i): & 2 i z=y-x, & (1,-1,-i): 2 i z=x-y, \\
(-i, \quad 1, \quad 1): & 2 i x=y+z, & (i, \quad 1,1):-2 i x=y+z, \\
(1,-i, \quad 1): & 2 i y=z+x, & (1, \quad i, 1):-2 i y=z+x, \\
(1, \quad 1,-i): & 2 i z=x+y, & (1,1, \quad i):-2 i z=x+y .
\end{array}
$$

3. The undulations of $Q$, as well as the tangents there, are all obtainable by linear construction when $q$ is given. The six lines on which the undulations lie pass two through each vertex of $\Delta$; the two through $y=z=0$, for example, are $y^{2}+z^{2}=0$ and these are harmonic 
not only to the two sides of $\Delta$ but also to the two lines, $y^{2}-z^{2}=0$, which join this vertex of $\Delta$ to those two vertices of $q$ which are joined by the opposite side of $\Delta$. So that we have the following construction:

Take any quadrilateral $q$ and its diagonal triangle $\Delta$. Through a vertex $X$ of $\Delta$ there pass two sides $X Y, X Z$ of $\Delta$ and two lines which join $X$ to the two vertices of $q$ on $Y Z$; there is a third pair of lines through $X$ harmonic to both these pairs. In this way there arise three pairs of lines, one pair intersecting at $X$, one at $Y$ and one at $Z$. The 12 intersections, other than $X, Y, Z$, of these six lines are undulations of a quartic curve.

Next: any undulation lies on two of the six lines, and they join it to two of the vertices of $\Delta$; the tangent at the undulation is, in fact, the harmonic conjugate, with respect to these two lines, of the line which joins the undulation to the third vertex of $\Delta$. For example: the undulation $(1, i, 1)$ is common to $y=i z$ and $x=-i y$; the join to the remaining vertex of $\Delta$ is $i(y-i z)-(x+i y)=0$, whereas $i(y-i z)+(x+i y)=0$, or $z+x+2 i y=0$, is the line which has been seen to be the tangent of $Q$ at the undulation $(1, i, 1)$.

It also happens, and can at once be verified by using their coordinates, that four collinear undulations form a harmonic set, and that the two harmonically conjugate pairs have for their common harmonic pair that constituted by the vertex of $\Delta$ on the line and the intersection of the line with the opposite side of $\Delta$.

4. The inflections of a plane curve all lie on its Hessian, and when the curve is without singularities, as $Q$ is, the inflections constitute the whole set of intersections of the Hessian with the curve. When two inflections coalesce in an undulation the Hessian must not merely intersect the curve at the undulation but must touch it there. It must therefore happen that the Hessian of $Q$ touches $Q$ wherever it meets it.

The Hessian of $\Sigma$ may be taken as

$$
\begin{aligned}
H & \equiv\left|\begin{array}{ccc}
2 x^{2}+y^{2}+z^{2} & 2 x y & 2 z x \\
2 x y & x^{2}+2 y^{2}+z^{2} & 2 y z \\
2 z x & 2 y z & x^{2}+y^{2}+2 z^{2}
\end{array}\right| \\
& \equiv 2\left(x^{2}+y^{2}+z^{2}\right)^{3}-3\left(x^{2}+y^{2}+z^{2}\right)\left(y^{2} z^{2}+z^{2} x^{2}+x^{2} y^{2}\right)+5 x^{2} y^{2} z^{2}
\end{aligned}
$$


It is seen at once that $H$ vanishes at each of the 12 undulations of $Q$, and it is an easy matter to verify that, at each undulation of $Q$, the curve $H=0$ has the same tangent as $Q$. But these facts can also be established otherwise. For the curve

$$
\left(y^{2}+z^{2}\right)\left(z^{2}+x^{2}\right)\left(x^{2}+y^{2}\right)=0
$$

consists of the six lines on which tetrads of undulations lie, and is a sextic curve with a node at each of the 12 points. It would therefore follow that, if $H=0$ were to touch $Q$ at each undulation, a member of the pencil

$$
H+\mu\left(y^{2}+z^{2}\right)\left(z^{2}+x^{2}\right)\left(x^{2}+y^{2}\right)=0
$$

would contain $Q$ as a part; and conversely. Moreover, since each sextic of the pencil is invariant under $G$, the conic which makes up with $Q$ the degenerate curve of the pencil must also be invariant for $G$, and so must be $C$. And indeed it happens that

$$
H+5\left(y^{2}+z^{2}\right)\left(z^{2}+x^{2}\right)\left(x^{2}+y^{2}\right) \equiv 2\left(x^{2}+y^{2}+z^{2}\right) \Sigma .
$$

It might also be remarked that each of the conics

$$
2 x^{2}+y^{2}+z^{2}=0, \quad x^{2}+2 y^{2}+z^{2}=0, \quad x^{2}+y^{2}+2 z^{2}=0
$$

contains four of the undulations and touches $Q$ at each of them; the three conics between them account for all the undulations, so that the sextic curve

$$
\left(2 x^{2}+y^{2}+z^{2}\right)\left(x^{2}+2 y^{2}+z^{2}\right)\left(x^{2}+y^{2}+2 z^{2}\right)=0
$$

also touches $Q$ at every undulation and must therefore belong to the above pencil. And, in fact,

$H+4\left(y^{2}+z^{2}\right)\left(z^{2}+x^{2}\right)\left(x^{2}+y^{2}\right) \equiv\left(2 x^{2}+y^{2}+z^{2}\right)\left(x^{2}+2 y^{2}+z^{2}\right)\left(x^{2}+y^{2}+2 z^{2}\right)$.

Mathematical Institute,

The University, Edinborgh. 